\title{
7 The influence of local ownership and politics of the use of evaluations in policy making
}

The case of the public procurement evaluation in Uganda

\author{
Ismael Kawooya, Timothy Lubanga, Abdul Muwanika, \\ Edwin Muhumuza and Rhona Mijumbi-Deve
}

\section{Summary}

This case study sought to understand the mechanism leading to the use of evidence from an evaluation of public procurement systems in Uganda undertaken in 2012-13, led by the Office of the Prime Minister (OPM) and the Public Procurement Development Authority (PPDA). The public procurement sector had undergone major changes over the years. The impetus for the evaluation was the need to improve the effectiveness and efficiency of the system, and was agreed to by Cabinet and supported by the World Bank through its Technical Advisory Support Unit. An independent consultant undertook the evaluation, working closely with the PPDA.Various structures oversaw the evaluation, including OPM and the Evaluation Sub-committee. The consultant was responsive to government requests for advice and maintained regular communication, reporting on a monthly basis. Ultimately, this resulted in an evaluation that was owned by government and seen to be of high quality. The evaluation led to revision of procurement thresholds and flexibility for sectors that need specialised procurement. It demonstrated to the PPDA the importance of regularly reviewing and updating regulations, standards and guidelines.

\section{Background}

Uganda has established mechanisms within government to improve the quality of policies through the use of evidence. OPM has, since 2005, led efforts to strengthen the national Monitoring and Evaluation (M\&E) system, including setting up a national evaluation system with an Evaluation Sub-Committee (ESC) and a Government Evaluation Facility (GEF), M\&E units in each government ministry, department or agency (MDA), and building their M\&E capacity. To date, over 30 evaluations have been conducted with various inputs into policies using this system. However, little is known of how these evaluations have actually been used in policy making. 
This case study focuses on evidence from an evaluation carried out in 2012 to assess and learn of the impact of public procurement reforms. The evaluation was completed after the laws amending the Public Procurement and Disposal (PPD) Act had been assented to by the president but before regulations and standards were enacted. Although the process for amending this Act had begun, not enough was known about how the previous law performed and it was felt to be necessary to understand how the current law might work. This prompted a request for an evaluation into the effectiveness of public procurement reforms in Uganda. This case sought to understand how the evidence from the evaluation was used during the amendment process of the PPD Act and the factors influencing its use. The case draws out lessons on how evidence might be used in similar settings in other countries in Africa. It specifically focuses on the complex relationships and interplay between the actors, institutions and processes within public procurement and how these affected the use of evidence in this process.

The research was undertaken from October 2018 to August 2019 using qualitative data collection methods and analysis, including document review and semi-structured interviews. Documents reviewed included both published and unpublished documents. Semi-structured interviews involved key informants from (1) government institutions: OPM, Ministry of Finance, Planning and Economic Development (MoFPED), Uganda National Roads Authority and PPDA; (2) the donor community; and (3) an evaluator representative. The participants interviewed were identified from stakeholder lists included in the appendices of evaluation reports and snowball sampling. Of the 12 potential interviewees, nine responded and were interviewed.

\section{The context}

\section{Background to Uganda}

The Government of Uganda outlines key strategic priorities for development every five years in a National Development Plan (NDP). The current NDP II introduces an ambitious goal, Vision 2040, which sets out strategies to transform Uganda's economy to middle-income status by 2040 through infrastructure development in energy, oil and roads (European Commission et al., 2015; National Planning Authority \& National Planning Authority, 2009).

Uganda receives significant budget support from development partners (DPs), and the World Bank is the biggest contributor, with a contribution estimated at 4.8\% of GDP in 2012/13 (European Commission et al., 2015). As such, the interests of DPs have had significant influence on the public financial management reforms undertaken by the government.

Critics often point out that, despite apparently good policy frameworks, implementation is lacking. For example, the World Bank's Country Policy and Institutional Assessment showed that Uganda scored an average of 3.6 against the average score of 3.1 in sub-Saharan Africa. ${ }^{1}$ However, on accountability, transparency and corruption in the public sector, Uganda scored a miserly 2.0 against a sub-Saharan Africa average of 2.7 (World Bank \& Government of Uganda, 2004). 


\section{The institutional context to the procurement sector}

An estimated $60 \%$ of the country's budget is spent on public procurement, which is comparable to a range of $40 \%-70 \%$ spent by developing countries (Khi V. Thai, 2009). Public procurement is organised in Uganda through the PPDA Act 2003, which defines procurement as 'acquisition by purchase, rental, lease, tenancy, franchise, or any other contractual means, of any type of works, services, or supplies or any combination' (Government of Uganda, 2003).

Public procurement is decentralised, meaning that central and local government entities are responsible for their own procurement processes from planning to implementation (Government of Uganda et al., 2003; Procurement and disposal of assets authority, 2004, 2005). Public procurement processes with government MDAs are managed through a procurement cycle at the procuring and disposing entity (PDE), which includes a user department that initiates the process to procure a service or good, and a procuring and disposal unit (PDU) that coordinates the administrative process. An accounting officer designated by the secretary to the Treasury is responsible for the accountability of funds disbursed to the PDE.

A number of different surveys have concluded that corruption in the government is an acknowledged way of 'doing' business (Procurement and disposal of assets authority, 2011). The World Bank estimated that Uganda lost about USD 500 million to corruption annually before 2011 (Procurement and disposal of assets authority, 2011). During the OPM and the Karuma dam scandals in 2009 and 2002, DPs withdrew and/or froze aid to the government (Harold, 2012; Michael, 2002; Walubiri, 2012).

\section{Influential actors and stakeholders}

\section{State actors and stakeholders}

The president of Uganda is a leading proponent of public sector reforms and has on several occasions made political proclamations that set the precedent for reforms (Nabyonga-Orem et al., 2014).

Public financial management (PFM), including procurement, falls under the MoFPED, which is responsible for policy formulation, while the PPDA has been established as an autonomous regulatory body that regulates and monitors compliance to the PPDA Act by all government ministries, departments and agencies (MDAs). The MoFPED's Procurement Policy and Management Department is responsible for managing and coordinating public procurement reforms through initiation of public procurement policy reviews.

The PPDA Authority is mandated by the PPDA Act to regulate and monitor compliance and performance and ensure the necessary capacities are in place for public procurement. The Authority recruits and supports procurement officers for all government entities, ensuring interaction between the regulator and implementing entities through capacity building (Government of Uganda, 2003). The PPDA Authority has a research unit and has been involved in a number of research activities to improve the efficiency and effectiveness of procurement reforms. Structural mechanisms are established to ensure that 
the reports from the research unit are discussed and decisions are made by the Procurement Performance Monitoring System.

The OPM coordinates and implements the strengthening of the national M\&E system. The Evaluation Sub-Committee (ESC) is an initiative within OPM to support public sector evaluations through technical guidance and is composed of experts from development partners (DFID and the World Bank), OPM, MoFPED, Uganda Bureau of Statistics and the Economic Policy Research Centre at Makerere University. The topics for evaluation are identified by the permanent secretaries or directors of respective government entities, presented to Cabinet, and discussed in the Joint Budget Support Framework (JBSF). Engaging Cabinet ensures that there is high-level buy-in from government. The prioritised list of evaluations is then agreed on and forwarded to the ESC. The purpose, methods and finances of the evaluations are discussed within the ESC.

I think the public sector M\&E policy is also important because in that policy, we (OPM) articulate requirements, for example, that evaluations should be independent; therefore, we procure evaluators for the public sector; therefore, we are required to follow the necessary laws and regulations concerning procurement.

(Respondent 7 - Government)

As the system is decentralised, all government entities have discretion over their own procurement. Sectors with unique practices (such as engineering, roads and health) have separate procurement standards and regulations. These institutions are able to instigate reforms, in consultation with PPDA, to ensure that the necessary procurement regulations and guidelines are in place.

\section{Non-state actors and stakeholders}

Services and goods are provided to MDAs by the private sector under the guidance of the PPDA.These providers can influence the procurement process through lobbying the MDAs or the PPDA for more flexible policies and standards in accountability, corruption and fraud.

Civil society organisations such as non-governmental organisations, faith-based and community groups are instrumental in presenting community opinions on corruption and malpractices in public procurement. They do so under the umbrella of the Anti-Corruption Coalition Unit as well as other laws, such as the Access to Information Act, 2005, that provide for the right to access of information.

Communities also have an influence through community barazas, which were started by OPM in 2009. These are open events, organised by local governments, at which district service providers report on progress and the public is able to discuss issues related to implementation of services and infrastructure in their area. The PPDA also uses them to provide and receive information on specific procurement processes.

Those Barazas ... are holding the accounting officers accountable. PPDA is presenting those findings to civil society organisations that are making noise when those contracts are not made.

(Respondent 6-Government) 


\section{Development partners}

DPs providing substantial budget support through the Poverty Eradication Action Plan and Poverty Action Fund include the World Bank, DFID, African Development Bank (ADB), United Nations Development Programme (UNDP), United States Agency for International Development (USAID), and Deutsche Gesselleschaft für internationale Zusammenarbeit (GIZ). DPs are particularly interested in how their funds are spent, as they have to report to their respective country's taxpayers. Consequently, they have a high interest in financial accountability and transparency of funds, which led to sustained pressure to institute public financial management reforms, including those on procurement. DPs also support evaluation through providing funding to evaluations, now formalised through pooled funds under the GEF.

They (DP) have their own rules and guidelines ... they have the financing agreements - they can make certain policy decisions. So, they (DP) have had a huge influence on public procurement in this country - ADB, World Bank and the like.

(Respondent 5 - Government)

\section{The policy timeline}

The public finance (Tender Board) regulations of 1977 were passed under the 1964 Public Finance Act, which replaced the colonial system where public procurement for most government entities was conducted through a central tender board (Dza et al., 2013; KhiV.Thai, 2009; Sabiiti and Muhumuza, 2012). However, the promulgation of a new constitution in 1995 expanded the size of government:

The first thing that happened was the decentralisation policy, the many constitutional bodies got created, then acts of parliament created many agencies, privatisation, so many regulators.... So it was not feasible to continue with ... sending requests to a certain unit in the Ministry of Finance to procure a phone, a car, building. It was just not possible anymore.

(Respondent 5 - Government)

In 1997, a national task force set up by the MoFPED affirmed that the centralised procurement model had outdated regulations and procedures and was fraught with inefficient and fragmented processes, corruption and fraud. The National Task Force made four recommendations for public procurement: drafting and enacting a legal and regulatory framework for public procurement, decentralising public procurement to the procuring entities, establishing a statutory autonomous body to set rules, monitoring procurement and review complaints, and harmonising procurement policies and practices at both central and local levels of government.

In 2001 the public (procurement) finance regulation was enacted, dissolving the Central Tender Board (Khi V. Thai, 2009; Sabiiti and Muhumuza, 2012). In the same year, the World Bank supported the first Country Procurement Assessment Review 2001 (CPAR) to benchmark key performance criteria and 
the organisation of a public procurement system, which formed the basis of establishing indicators for M\&E within the sector. This culminated in the passing and enactment of the Public Procurement and Disposal of Public Assets Act, 2003, regulations, guidelines and policies (Act 1 of 2003) (Government of Uganda et al., 2003), which provided for the establishment of an autonomous body known as Public Procurement and Disposal of Public Assets Authority (PPDA). The Act prescribed the objectives, functions and powers of the Authority, but without provisions for local governments. Therefore, in 2006, the Local Government Act was amended to include these.

In 2009, the government initiated the amendment of the PPDA Act 2003 to include the Local Government Act amendments which was passed by Parliament in 2011. The regulations, standards, guidelines and circulars were completed in 2014 and the Act finally enacted.

\section{The evidence journey}

In 2010, priority topics for evaluation had already been decided on between the government and DPs, and the amendments to the PPDA Act of 2003 were underway. Concerns were raised that the amendments did not address the challenges that had previously existed in procurement. The World Bank, in particular, argued that there was a need for an evaluation of the procurement sector to better understand these challenges. The evaluation was commissioned in 2011 by the Government of Uganda through the OPM, with technical assistance from the Technical Administration Support Unit (TASU) of the World Bank and funding from the World Bank and DFID. In October 2012, an Indian consulting firm was contracted by TASU through open tender to carry out the evaluation.

The purpose of the evaluation was to assess the effectiveness of the PPDA Authority on procurement practices in the public sector, with particular focus on the roads and energy sectors. The evaluation had three specific objectives:

- To assess the effectiveness, efficiency and sustainability of procurement reforms/interventions undertaken in Uganda since 2003

- To identify lessons learnt and provide recommendations for informing future PFM interventions in public procurement

- To draw lessons learnt from both intended and unintended results and propose solutions/measures to provide sustainability of successes realised so far.

A three-level management system was set up to provide support and oversight to the consultants during the evaluation - a management committee, a reference group and an evaluation sub-committee to provide support and guidance. These included technical experts in procurement and evaluation from PPDA and OPM. The steering committee played the oversight role, together with TASU. 


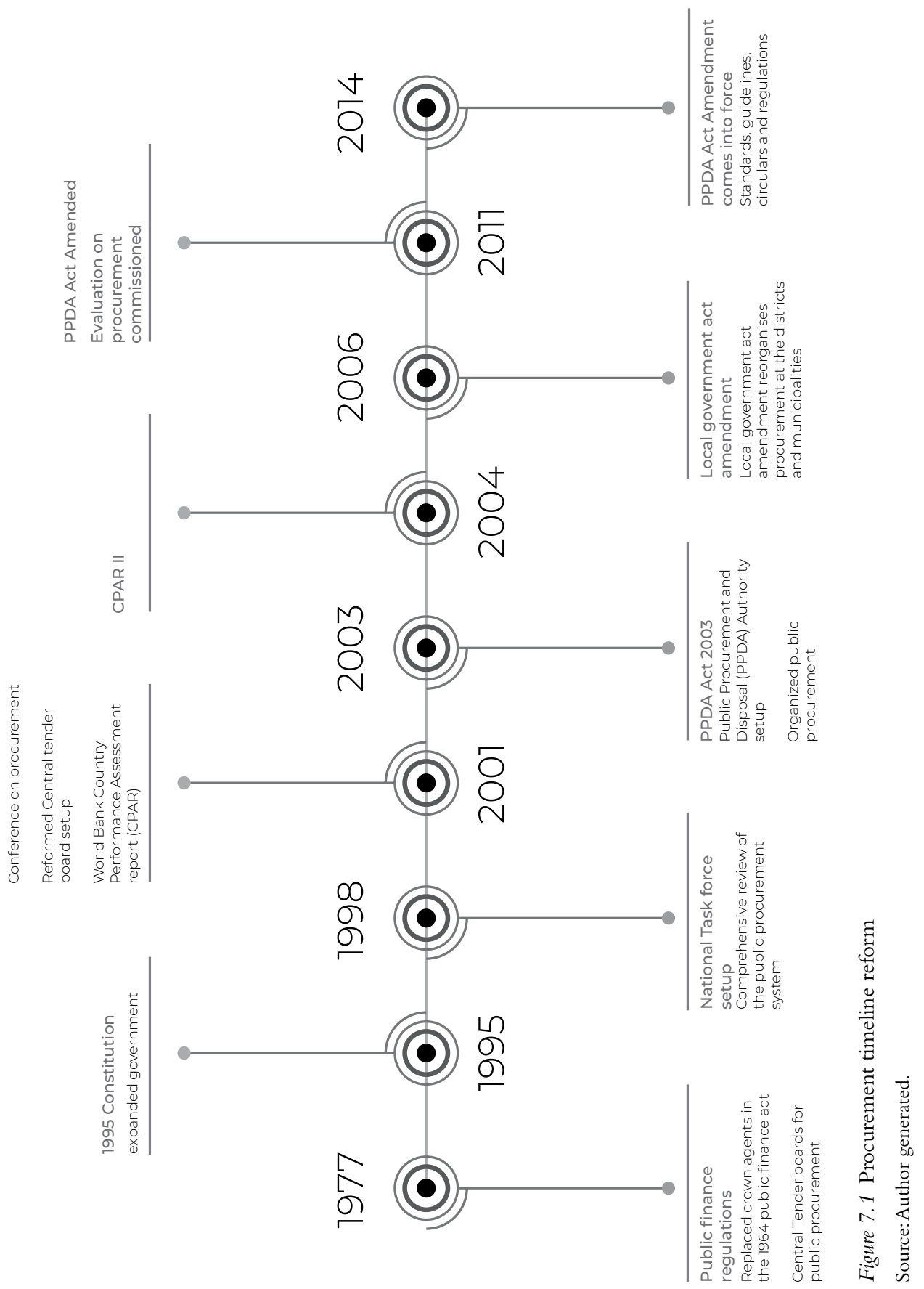


The evaluation was initiated with a scoping mission, during which the consultants met with key stakeholders. Of significance was ensuring buy-in from the PPDA Authority as the lead government agency in the implementation of the evaluation. This was done through meetings and personal contact between the consultant and officials from OPM and the PPDA Authority. The Authority identified and delegated a contact person to act as liaison for the consultant, and office space was provided.

At the beginning, the consultants were invited to comment on the amendment to the PPDA 2003 before it was passed (such as on definitions and clarifications where confusion might ensue), as the amendment process was already underway.

Now if PPDA Authority did not agree or was not on board in terms of the purpose/ usefulness of this evaluation ... then whatever came out would not be taken on board that easily. That is ... the consultants were presenting their findings every week and these players were picking out what mattered to them.

(Respondent 3 - Government)

The consultants conducted an initial assessment to understand the context of public procurement in Uganda, from which an inception report was drafted and commented on by the steering committee and the PPDA Authority. They then proceeded to implement the evaluation, keeping the steering committee and TASU appraised of the field findings on a monthly basis. The final report was drafted and approved in 2013, commented on, discussed and revised with guidance from the steering committee and the PPDA Authority. ${ }^{2}$

Overall, the evaluation was received positively.The OPM's M\&E department considered the evaluation to have had a rigorous methodology and findings were robust.

We rate this as one of the very good evaluations undertaken - done properly - satisfied with the quality of results. It was international competitive bidding-firm from India became the best. The evaluation sub-committee approved the evaluation report with many comments but were satisfied with the final product.

(Respondent 8 - Government)

\section{Using the findings of the evaluation}

In this research, use has been categorised as conceptual, instrumental, process or symbolic (see Chapter 2). Instrumental use of evidence involves responding to specific findings or recommendations, while conceptual use is generally when the evaluation is used to enlighten, influence, inform or clarify a policy issue indirectly (Amara et al., 2004). Negative symbolic use involves applying research results to legitimise or support an already determined decision or 
policy (Amara et al., 2004), while positive symbolic use increases the profile of the sector or topic.

In terms of instrumental use, the evaluation report had specific recommendations that were adopted in the guidelines, standards and circulars passed by Parliament in 2014, including:

- Revision of procurement thresholds for different bidding methods and implementing a system to update these;

- Flexibility for sectors that need specialised procurement, for example, roads, electricity and health;

- The solicitor general's approval was required for all procurement above UGX 50 million but this created unnecessary delays, especially in sectors that deal with huge budgets (e.g. construction). This was revised to above UGX 200 million but there is still contestation that this is too low for some sectors.

The PPDA Authority was in the middle of revising the Act and the regulations - this evaluation enriched the argument for the revision of the Act and regulations (Key informant 8 ).

In terms of conceptual use, the evaluation demonstrated to PPDA the importance of regularly reviewing and updating regulations, standards and guidelines. Government had undertaken the amendment of the PPDA Act without reviewing its effectiveness and efficiency. Indeed, it can be argued that the evaluation also influenced the use of evidence in the revision of the law in the public procurement sector. This can be shown with the current amendments for the procurement law that are considering using the evaluation as a blueprint for the next evaluation. The evaluation was also reported to have had a significant effect on the conduct of evaluations in Uganda and has been incorporated into the curriculum of students learning about evaluation.

\section{Understanding factors influencing use of evaluation}

\section{Demand for evaluation}

The evaluation priorities had already been identified and did not include procurement, while the amendment process of PPDA 2003 was already underway (Respondent 8 - Government). It is unlikely that the evaluation would have taken place without pressure from the World Bank and the influence DPs had on government because of the budget support they provided. In addition, there was broad agreement among key players about the complexity of the challenges that the sector had experienced in the past and the need to gather evidence and insights into the underlying reasons behind these.

It is also important to note that while pressure from the DPs was the key driver behind demand, the existence of an evaluation culture with well-developed 
frameworks and mechanisms provided a mechanism for demand to be implemented, including:

- The existence of a National Evaluation Policy and Board with requirements for and guidance on evaluation;

- Process of identification and prioritisation of evaluation topics involving and driven by leadership within government (permanent secretaries or directors of respective government entities and Cabinet);

- The JBSF, comprising key government institutes and development partners and providing a platform for discussion and influence on prioritisation of evaluation topics.

\section{Interventions that promoted and ensured use}

This section hypothesises mechanisms between the approaches and interventions used prior, during and after the evaluation and how this affected the use of evaluation findings and recommendations. These are summarised in Table 7.1 and used to inform lessons and reflections in the final section.

Table 7.1 Use interventions and their effect

\begin{tabular}{ll}
\hline Use intervention & Effects and change mechanisms activated enabling use of evaluation \\
\hline $\begin{array}{l}\text { National M\&E Policy and } \\
\text { National Evaluation } \\
\text { Board }\end{array}$ & $\begin{array}{c}\text { Provided requirements for and guidance on evaluation to } \\
\text { ensure credibility of evaluations }\end{array}$ \\
$\begin{array}{l}\text { Independence of } \\
\text { implementing agency } \\
\text { from beneficiary agency }\end{array}$ & $\begin{array}{c}\text { On setting up evaluation, it was decided that an independent } \\
\text { agency oversee it to minimise bias in the event of conflicts } \\
\text { of interest. The OPM was the implementing agency } \\
\text { and the beneficiary agency, PPDA, provided guidance } \\
\text { throughout the evaluation. This was pivotal to the eventual } \\
\text { agreement with evaluation results, and so likely to be used. }\end{array}$ \\
$\begin{array}{l}\text { Provided clearly defined mechanisms for collaborative } \\
\text { identification and prioritisation of evaluation topics involving }\end{array}$ \\
levaluations and JBSF \\
of government and DPs. This enabled agreement between key \\
actors such as OPM and PPDA Authority in particular on \\
the need for evidence to better understand and address policy \\
challenges within the public procurement sector.
\end{tabular}


A key factor identified as influencing use was the extent to which there was a sense of ownership of the process and findings. Ownership can be affected by multiple things: drivers behind demand; ensuring a shared agreement and understanding around the purpose, objectives and methods; the entity commissioning the evaluation (particularly if different from end users), the extent to which political and technical leadership is involved, the way in which consultations and discussions are carried out and so forth.

One of the challenges was that ownership question. Once these evaluations are decided by somebody outside and that person manages the funds and procures and just sends the person, you rarely find the kind of ownership needed to implement some of these things. Yes, I might have been involved at my level, but we tended to sense that you are briefing the owner (really) like a second party. If an evaluation is being done on impact of procurement reforms, the Executive Director and the highest level should really be at the centre of it all. But if they are being briefed - it is like me coming to tell you stuff about your house. You are listening to it like I should be knowing better. That ownership question still has to be answered.

(Key informant 5)

Table 7.1 shows the use interventions that played a role with this evaluation. Many of these arise from the emerging national evaluation system and reflected the need for credibility and ownership.

\section{Barriers and enablers to evidence use}

This section identifies and reflects on some of the factors in the wider context which influence use of evidence.

Abilities to understand and navigate the realities of the wider political and social environment

Policy making is a political process that considers the interests of diverse groups. These interests have to be weighed with the greater good by policy makers, taking into consideration factors such as ethics, culture, costs and politics. Whereas Uganda has had a multiparty political system for 13 years, the ruling party has been in power for 30 years. Policies for politically sensitive sectors are often made in favour of the party in power, regardless of the available evidence. Some respondents indicated that politically sensitive sectors are cautious of how they use evidence, as their decisions may be overridden by political decisions.

Of course, you can't do away with political interference/workmanship because these are political decisions. Evidence is only used to inform these decisions. You need to be aware of the political situation in the country and if it is considered politically sensitive - for example, the agriculture 
evaluation was so politically sensitive. This means that the evidence would still get disregarded.

(Respondent 2 - Non-government)

Political events such as elections, strikes or protests often create environments in which individuals are sensitive to sharing information that may be used against them. Another factor influencing willingness of individuals to provide information is perceived risks associated with confidentiality. In the case of a sector such as procurement that has multiple players, it is easier to trace information back to individuals at the district level than at the national level. This, in turn, created challenges for ensuring that evidence gathered was comprehensive and accurate, which is necessary to ensure credibility and therefore use.

Familiarity with the context is key for findings to be relevant and recommendations feasible.

It was done by foreigners from India. . . . They did very well in terms of their competencies but . . . all recommendations should have context. You must ... understand all the different social, political and technological context you are dealing with.

(Respondent 5 - Government)

The timing as well as time taken to undertake and finalise an evaluation are important in this regard. This evaluation took two years to complete which may have possibly affected the quality of the discussions during the policy process.

If, for example, you do an evaluation on energy towards a general election, you will not get a lot of evidence. They will even say why are you doing it at this time when we are going for general elections. So, the timing becomes critical. For example, in this case the PPDA had been in place for some number of years and maybe the findings of the evaluation would trigger the need for a mini or comprehensive review of the Act because much as it was focusing on the two sectors, the principle would cut across. (Respondent 3 - Government)

National systems and processes guiding evidence

At the national level, there are well-established systems for $\mathrm{M} \& \mathrm{E}$ in the public sector, such as the GEF at the OPM, discussions of performance monitoring reports from the OPM, M\&E unit by the Cabinet Secretariat and so forth. Another is the regulatory impact assessment guidelines used by policy makers when considering a policy review (Ministry of Finance Planning and Economic Development, 2004).

\section{Influence of DPs}

DPs were influential in evidence use, both positively and negatively. On the one hand, their interest in an environment with proper fiduciary mechanisms and level of influence was instrumental in ensuring that the evaluation was 
commissioned and used. On the other hand, some respondents spoke about donors pushing their own agendas that might not necessarily be in the best interest of the host country (such as the structural adjustment reforms). In addition, in a situation with limited trust between the host and the donors, the host country might simply agree to an evaluation supported by the donor to appease the donor. However, this often leads to a lack of ownership with the report being shelved and findings not even discussed.

The World Bank was interested in procurement and paid for the evaluation. There was a lot of push from the World Bank during the process. This could have introduced bias.... But I do not think it should matter because procurement is very important.

(Respondent 2 - Non-government)

\section{Cultures, systems and capacities}

Decision-making structures within the PPDA Authority established a culture in which evidence is reflected on. These structures consider evidence generated through reports from PDEs, audit reports or the government procurement portal and use evidence at different managerial levels. The PPDA has a research unit responsible for gathering evidence about public procurement through monthly reports to the Authority, investigations and audits, in addition to coordinating with research institutions to conduct relevant policy research (e.g. integrity surveys). The reports from the evidence-gathering activities are discussed through structural mechanisms established within PPDA, such as meetings and presentations at each hierarchical administrative level up to the board, so that decisions can be made.

It has been argued that organisations with mechanisms for generating evidence are more likely to build a culture that values the use of evidence within policy making (Goldman, 2018). This was the case with PPDA, which established the PPMS in 2009 as a mechanism for regular gathering of quantitative data. The data collected is summarised and reported on a monthly basis and these reports are shared with the MoFPED, which initiated procurement policies.

The capacity of policy makers to use evidence was raised, and that policy makers' background influences their ability to interpret evidence. Policymakers should understand the value of evidence in policy decisions and how to interpret it to motivate them to use the evidence.

\section{Leaders and champions}

In this evaluation, for example, individuals in leadership positions pushed for a stronger $M \& E$ function in all government programmes, such as the former permanent secretary in the OPM and the prime minister, championing the use of M\&E evidence.

The PS at the time had just started and thought this was a difficult job. He then looked at what he could use as leverage and discovered that the OPM was responsible for coordination of government business. He empowered 
OPM. He looked at the mandate and wondered how you do that. So, there he looked at evaluations.

(Respondent 2 - Non-government)

The experience of the procurement sector showed that adequate and comprehensive stakeholder consultations positively influence the use of evidence in policy-making processes. Engaging enough stakeholders increases the opportunities for evidence to be used in the decision-making processes. During the engagement processes, some of these stakeholders might champion the use of evidence or at times use the evidence directly for policy decisions.

Interview respondents also identified risks associated with the use of evidence by influential individuals. For example, policy makers interested in pushing their own agenda may use the evidence to support an already predetermined position (an example of negative symbolic use).

\section{The nature of the evidence itself}

Interview respondents also noted that the reliability of data (actual or perceived) affects how the evidence is used in the policy-making process. Government data is often incomplete or inaccurate leading to lack of trust by policy makers in the data or the sources (Respondent 5 - Government).

Equally important is how evidence is communicated. Researchers need to ensure that it is packaged in an accessible format and easily obtainable and understood. This includes considerations around language, accessibility or media. Evidence and recommendations also need to be specific, providing clarity in findings and guidance. The more generic, the less likely they will be used.

\section{Reflecting and learning from experience}

\section{How the context and intervention influence the use of evidence}

Context is key, and in that particular context, which mechanisms have led to what outcomes, which we summarise as the Context-Mechanism-Opportunity configuration. We take the mechanisms to include the actual evidence generation, interventions applied to promote use and change mechanisms (such as building agreement or awareness) that these use interventions sought to achieve. The immediate outcomes were changes in capability, opportunity or motivation to use evidence, while the wider outcomes were actual changes in policy or practice.

Key elements in the wider context in this case study were a fairly wellestablished national evaluation system which provided a framework for evaluations required and informed decision-making processes. There was also a recognised need for reforms within the procurement sector and pressure from the World Bank to undertake the evaluation to support the reform process. The evidence generation process was felt to be robust and credible, carried out by 
a well-qualified independent consultant guided by evaluation clients and the beneficiary agency, PPDA.

Mechanisms critical to ensuring use of the evaluation findings included processes and structures that enabled ownership and trust in the process. These included the use of committees for oversight and technical guidance, involvement of stakeholders and credible consultants at all stages. In addition, regular meetings with the client, beneficiaries and wider stakeholders strengthened awareness, confidence and the ability of stakeholders to engage with the evaluation process and findings. The quality of the evaluation and trust in the process ensured the motivation to utilise findings, and the sector reform process created the opportunity to do so.

Ultimately, this resulted in revision of the Act and regulations (i.e. instrumental use of the evaluation findings). In addition, the evaluation demonstrated the value and importance of evidence in decision making and is informing the conduct of evaluation in the country.

\section{Lessons for the country and beyond}

\section{Ensuring independence}

For a sector such as procurement, with significant levels of funding and affecting multiple other sectors and stakeholders, the level of independence, real or perceived, is critical to maintaining a sense of credibility and trust, and therefore ensuring use of evaluation findings.

In this case, independence was maintained by a separation of roles between the agencies commissioning, implementing, and the ultimate users of the evaluation. The organisational structure of the evaluation provided the process with independence from political interference, which also enabled the evaluation team to engage with respondents at ease. Some of the key features of this structure included agreement on purpose and relevance of the evaluation by key decision makers in Cabinet and the JBSF, while PPDA's role was primarily to provide technical guidance, ensuring that evaluation was aligned with the sector policy needs, providing feedback, commenting on reports and so forth.

\section{Ownership}

Across many countries in Africa, the role and influence of DPs is significant as a result of budget and other support. In situations where DPs are involved in evidence generation and use, attention needs to be paid to ensuring local ownership of the process and findings.

This case study demonstrated the importance of an evaluation process that the PPDA considered its own. The stakeholders had preparatory meetings with GPCL consultants, chaired by the executive director of the PPDA. A liaison officer was selected as the PPDA contact person to work with the 
consultants and office space was provided for them by the PPDA. In initial meetings, stakeholders agreed on the purpose of the evaluation and way forward. There was constant interaction between consultants and the PPDA.The positive nature of the relationship was reflected by the invitation extended to the consultants to comment on the amendment before it was passed by Parliament. However, there was also a feeling that since the initiation of the evaluation came from the OPM and not the PPDA, the evaluation was imposed on the PPDA without significantly involving senior management. Overall, there was a positive effect on the openness of the Authority to the evaluation and willingness to utilise findings in the regulations, guidelines and standards in the amendment process.

\section{Credibility and trust}

Evidence that is not trusted and perceived as credible is unlikely to be used. Efforts to ensure credibility of the evaluation included using an independent agency to provide oversight (OPM), procuring a competent consultant for the task and ensuring rigorous and robust methods. The consultants undertook a scoping mission to understand the context, and baseline surveys on public procurement. During the evaluation, the consultant had monthly progress meetings with the evaluation sub-committee to update on progress and agree on a way forward. These meetings kept the stakeholders appraised of and provided valuable feedback to the evaluation process. In addition to the meetings, the consultant provided reports - inception, activity, scoping and final - that were commented on by the PPDA and evaluation sub-committee before being adopted as final reports. These efforts allowed the stakeholders to trust the findings from the evaluation and consider them during the amendment of the regulations, guidelines and standards.

Continuous feedback in the process is critical because it brings out the challenges that one is encountering during the evaluation process and that helps with coming up with strategies to address them before the final thing. But also, weekly feedback helps the stakeholder, the beneficiary, to know whether the evaluation is on track and likely to meet the purpose why it was initiated.

(Respondent 3 - Government)

\section{Capability}

Irrespective of how credible or robust an evaluation may be, it needs to be received within an environment that is capable of utilising the evidence. This capability is determined by culture, structures and processes, skills and technical experience.

The PPDA put in place structures and processes such as public procurement management system (PPMS) to continuously gather data and statistics 
on the state of public procurement and processes and to discuss these results at different managerial levels. The authority had also previously commissioned surveys, especially on corruption within the public procurement system, and had in place structures to discuss and consider the findings from all evidencegathering activities. The evaluation was jointly conducted by consultants and members of the PPDA, thereby increasing the PPDA's skills in conducting evaluations, as well as interpreting and communicating the evidence to policy makers.

\section{A final thought}

The Ugandan government has taken steps in institutionalising the use of evidence with structures and policies to support evidence generation, translation to policies, and implementation. The OPM has been at the forefront of driving the evidence agenda in government through the GEF and Evaluation Subcommittee and through chairing the JBSF. There is also a M\&E framework and regulatory impact assessment guide among policies that guide government entities in the implementation of evidence-informed policy making in their departments. However, in practice, evidence-informed policy making in Uganda is always going to be a challenge because many decisions are political and made without consideration of the evidence. This case study presents an opportunity to understand some of the reasons behind this and hopefully the insights generated may contribute to addressing the persistent challenges and barriers to use.

\section{Notes}

1 A weighted score that assesses a country's sustainable growth and reduction of poverty across 16 criteria. It has a minimum score of 1 and maximum of 6 .

2 Examples of bottlenecks and issues identified by evaluation include:

- Procurement thresholds have not been revised since 2003;

- The procurement plan is not systematically used as a monitoring tool;

- Absence of specialised standard bidding documents for infrastructure sector such as design and build, performance-based contracting;

- Delays in approval from contracts committee at almost all stages of procurement cycle;

- Delays in obtaining approval from the Solicitor General for contracts above UGX 50 million.

\section{References}

Amara, N., Ouimet, M. and Landry, R. 2004. New evidence on instrumental, conceptual, and symbolic utilization of university research in government agencies. Science Communication, 26(1), 75-106. https://doi.org/10.1177/1075547004267491

Dza, M., Fisher, R. and Gapp, R. 2013. Procurement reforms in Africa: The strides, challenges, and improvement opportunities. Public Administration Research, 2(2). https://doi. org $/ 10.5539 /$ par.v2n2p49 


\section{2}

Ismael Kawooya et al.

European Commission, Independent Evaluation Group, \& Government of Uganda. 2015. Joint evaluation of budget support to Uganda final report. Kampala: European Commission, Independent Evaluation group, Government of Uganda.

Goldman, I. 2018. Baseline on performance MEE culture in the public sector in Uganda, Benin and South Africa. Wits.

Government of Uganda, Ministry of Health, \& Ministry of Agriculture, F. and A.I. 2003. Uganda food and nutrition policy.

Harold, E.A. 2012. What lies beneath the corruption in the office of the Prime Minister? Daily Monitor.

Khi,V.T. 2009. International handbook of public procurement. 6000 Broken Sound Parkway NW, Suite 300 Boca Raton, FL 33487-2742: Taylor and Francis.

Michael, P. 2002. AES uncovers bribery in Bujagali Dam Project. NewVision.

Ministry of Finance Planning and Economic Development 2004. A guide to good regulation. In: PROGRAMME, R. B. P. (ed.). Kampala: Government of Uganda.

Nabyonga-Orem, J., Ssengooba, F., Mijumbi, R., Kirunga Tashobya, C., Marchal, B. and Criel, B. 2014. Uptake of evidence in policy development:The case of user fees for health care in public health facilities in Uganda. BMC Health Services Research, 14(1), 639. https:// doi.org/10.1186/s12913-014-0639-5

National Planning Authority, \& National Planning Authority. 2009. National development plan 2010/11-2014/15.

Procurement and disposal of assets authority. 2004. Annual report (Year Ended 2004). Procurement and Disposal of Assets Authority.

Procurement and disposal of assets authority. 2005. Report of the fifth procurement sector review. Workshop held on 29 June 2005 at Speke Report Munyonyo.

Procurement and disposal of assets authority. 2011. Annual report for financial year 2010/11. Kampala: Procurement and Disposal of Assets Authority.

Sabiiti, C.K. and Muhumuza, E. 2012. Second generation procurement. Moving from compliance to results in public procurement. Trends, challenges and opportunities from the Uganda experience. Presented at the International Public procurement conference, Seattle, USA.

Walubiri, M. 2012. Nsibambi regrets OPM scandal. NewVision.

World Bank \& Government of Uganda. 2004. Country procurement assessment report (CPAR). Kampala:The World Bank. 